\title{
Molecular characterization of fish neurolin: a growth-associated cell surface protein and member of the immunoglobulin superfamily in the fish retinotectal system with similarities to chick protein DM-GRASP/SC-1/BEN
}

\author{
Ute Laessing ', Suzanne Giordano', Brigitte Stecher ', Friedrich Lottspeich ${ }^{2}$, Claudia A.O. Stuermer ' \\ ${ }^{1}$ Faculty of Biology, University of Konstanz, D-78434 Konstanz, Germany \\ ${ }^{2}$ Max-Planck-Institute for Biochemistry, Genzentrum, D-82152 Martinsried, Germany
}

\begin{abstract}
We have used the polymerase chain reaction to isolate cDNAs coding for goldfish and zebrafish neurolin, a previously identified $86 \mathrm{kDa}$ cell surface glycoprotein in the goldfish visual system. Sequence analysis demonstrates that neurolin belongs to the immunoglobulin superfamily and is $51 \%$ similar to the chick cell adhesion molecule DM-GRASP, SC-1, BEN. Northern analysis with a riboprobe coding for the C-terminus of neurolin detected two mRNAs of $3.7 \mathrm{~kb}$ and $3.3 \mathrm{~kb}$ in both embryonic and adult goldfish.

Several monoclonal and polyclonal antibodies were generated against immunopurified goldfish neurolin and two are shown to crossreact with zebrafish proteins. Both antibodies identify a zebrafish protein of the same molecular weight as goldfish neurolin on immunoblots. Immunohistochemical studies with these antibodies in the zebrafish retinotectal system demonstrate labeling on young ganglion cells and growing retinal axons in a pattern similar to that found in goldfish. The similarity of neurolin to a known cell adhesion molecule, its expression on developing retinal ganglion cells and axons in both embryos and adult fish, and its re-expression during retinal axon regeneration in the goldfish suggests that neurolin is important during axonal growth in the fish central nervous system.
\end{abstract}

\section{Introduction}

Axons of the adult fish central nervous system (CNS) can regenerate following injury, unlike those of the mammalian CNS [10, 25]. Many molecules known to be involved in axonal growth and guidance in the developing nervous system are reexpressed in the adult fish CNS during regeneration $[5,29]$. We recently identified several growth-associated cell surface molecules in the goldfish visual system which are found on growing axons in the embryo and are reexpressed on axons in the retina

Correspondence to: C.A.O. Stuermer and optic nerve following optic nerve transection (ONT) $[1,21,29,32]$. Neurolin is an $86 \mathrm{kDa}$ glycoprotein of this group which was originally identified by the monoclonal antibody E21 [21]. In embryonic goldfish, neurolin is present on all retinal ganglion cells $(R G C)$ and their axons. In the adult, neurolin is absent from mature axons along most of their length, but is present on new and growing axons derived from the RGCs at the retinal margin [21] that, in adult goldfish, are continuously added to the retinal periphery [14]. Neurolin appears to remain on adult RGCs only at cell-cell contact sites [21] and is continuously found in the retinal axon terminal arbor layers of the adult tectum.

The reappearance of neurolin and other growth-associated cell surface molecules in the fish visual system following optic nerve lesions indicates that goldfish RGCs are capable of reexpressing molecules required for successful regeneration (reviewed in Stuermer et al. [29].

In the present study we have cloned and analyzed a cDNA coding for neurolin and demonstrate that neurolin is related to the recently discovered chick cell adhesion molecule (CAM) DM-GRASP belonging to the immunoglobulin (Ig) superfamily [2]; (the subsequently published chick cDNA sequences of $\mathrm{SC}-1$ [31] and $\mathrm{BEN}$ [22] are almost identical to DM-GRASP, therefore, for further comparisons, we will refer only to DM-GRASP). DM-GRASP shows a specific expression pattern in the chick embryonic nervous system and was shown to be a CAM capable of mediating axonal outgrowth and fasciculation in vitro [2]. The sequence similarities between goldfish neurolin and chick DM-GRASP, as well as the spatio-temporal expression pattern observed for neurolin in the embryonic and regenerating fish visual system indicate that neurolin may also be a CAM capable of facilitating axonal growth and guidance in vivo.

\section{Methods}

Animals. All goldfish (Carassius auratus) were maintained in tanks at $18^{\circ} \mathrm{C}$. Optic nerves of goldfish $(5-7 \mathrm{~cm}$ long) were cut under 
methanesulfonate salt (MS 222; Sigma, Dcisenhofen, Germany) anaesthesia. Adult zebrafish and embryos (Brachydanio rerio) were raised from a laboratory brecding colony and maintained at $28^{\circ} \mathrm{C}$. Embryos werc staged in hours postfertilization (h PF) [27].

Protein purification and amino acid sequencing. Neurolin was immunopurified over an antibody (monoclonal antibody E21) affinity column. The starting material was a pool of proteins solubilized by octylglucoside (OG) from a fraction enriched in cell surface membranes of adult goldfish brains [21]. For direct $\mathrm{N}$-terminal amino acid (AA) sequencing, the affinity-purified material was applicd to sodium dodecyl sulfate polyacrylamide gel electrophoresis (SDS-PAGE) and then analyzed after electroblotting onto Glassybond (Biometra, Göttingen, Germany) according to Eckerskorn et al. [7]. Intcrnal sequences were obtaincd by cleaving the protcin in the gel [6] with either protease LysC, trypsin or protease AspN (all enzymes from Boehringer, Tutzing, Germany), protein to enzyme ratio of $10: 1$ for $8 \mathrm{~h}$ at $37^{\circ} \mathrm{C}$. The peptides were eluted and separated by reversed phase high performance liquid chromatography (HPLC) on a Superspher 60 RP select B column (125x $2 \mathrm{~m}$ ), using a $0.1 \%$ trifluoroactic acid/acetonitrile solvent system (gradicnt: $0-60 \%$ in $40 \mathrm{~min}$; flow $500 \mu \mathrm{l}$; detection wavelength: $206 \mathrm{~nm}$ ). In one experiment the protein was digested with LysC dircctly in clution buffer of the affinity column adjusted to $\mathrm{pH}$ $8.5\left(8 \mathrm{~h}, 37^{\circ} \mathrm{C}\right)$; the enzyme to protein ratio was $1: 10$. To remove the detergent $O G$ the peptide mixture was chromatographed on a PolyLC Polysulfoethyl A cationic exchange column (ICT, Frank-

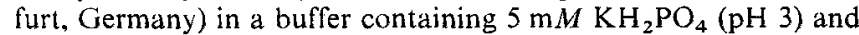
$25 \% \mathrm{CH}_{3} \mathrm{CN}$, applying a gradient to $90 \%$ of the same buffer containing $0.25 \mathrm{M} \mathrm{KCl}(40 \mathrm{~min}$; flow $300 \mu \mathrm{l})$. Pooled fractions were re-chromatographed on the reverse phase system given above. Amino acid sequence analyses were performed on a 477A pulsed liquid phase sequencer equipped with a 120A PTH analyzer (Applied Biosystems, Weiterstadt, Germany).

$R N A$ isolation and polymerase chain reaction. Twenty retinae were removed from goldfish 14 days following ONT and immediately frozen in liquid nitrogen. Poly $\mathrm{A}^{+}-\mathrm{RNA}$ was isolated with the Quick Prep mRNA Purification Kit (Pharmacia Biotech, Freiburg, Germany) according to the manufacturer's instructions. First strand cDNA primed with oligo dT18 was synthesized from $5 \mu \mathrm{g}$ of retinal mRNA using the Superscript Reverse Transcriptase (Gibco/BRL, Eggenstcin, Germany) in a $40 \mu \mathrm{l}$ reaction volume. One microliter of this reaction was used as a template for each polymerase chain reaction (PCR). Degenerate PCR primers, which include $5^{\prime}$ EcoR I sites, were synthesized corresponding to the neurolin AA sequence. Two initial primers were used: UL6 (sense) $5^{\prime}$ GGGAATTC.TTC/T.GAA/G.T/CTI.ACI.GTI.GAG/A.GG-3' and UL21 (antisense) 5'-CCGGGAATTC.T/CTT.A/GTG.A/ GTT.A/GTT.T/CTC.T/CTC-3' representing the amino acid scquences 'FELTVEG' and 'KHNNEE', respectively. The PCR reaction was initially denatured at $94^{\circ} \mathrm{C}$ for 2 min followed by 34 cycles of denaturing, $1 \mathrm{~min} 94^{\circ} \mathrm{C}$, annealing, $2 \mathrm{~min}$ at $45^{\circ} \mathrm{C}$, extension, 2 min at $72^{\circ} \mathrm{C}$.

The reaction produced a band of $\approx 500 \mathrm{bp}$ (pcrE21.1) which was cloned into the TA cloning vector pCR II (Invitrogen, San Diego, USA) as outlined by the manufacturer. The specific antisensc primer taken from the cDNA sequence of ncurolin, UL22 $5^{\prime}$-GGGAATTC.TTTAGGAATACCTTCCACCG-3' (containing a $5^{\prime} E c \circ \mathrm{R}$ I site) was used with the degenerate sense primer UL2 5'-TAC/T.GGI.GAA/G.ACI.ATT/A/C/G.T-3' to amplify the $1.1 \mathrm{~kb}$ fragment perE21.2 which was also subcloned into the $\mathrm{pCR}$ II-vector (Fig. 1). This reaction was run under the same conditions as above except that the annealing temperature was $41^{\circ} \mathrm{C}$.

To confirm the leucine (Leu) at AA position 325, four independent PCR fragments were amplified from goldfish cDNA template, using primer sets UL14/UL22 or UL29/UL22 (Fig. 1). The cloned fragments were subsequently sequenced in both directions.

All oligonucleotide primers were purified on sephadex NAP-10 columns (Pharmacia, Freiburg, Germany).
DNA sequencing. The cloned PCR bands were sequenced on both strands using a combination of internal sequencing primers and the M13 forward and reverse primers (Promega, Hcidelberg, Germany) in the vector. A deletion series was generated from clone pcrE21.2 using the double stranded Nested Deletion Kit (Pharmacia, Freiburg, Germany). The location of internal primers is shown in Fig. 1. Sequencing was done by the dideoxy chain termination method [24] with the T7 Sequencing Kit (Pharmacia, Freiburg, Germany) and the sequence was analyzed by the HUSAR sequencing system (Heidelberg Unix Sequencing Analysis Resources, release 2.0, DKFZ, Heidelberg, Germany).

The nucleotide sequences reported here were submitted to the Genbank database. The accession number for goldfish neurolin is L25056 and L25057 for zebrafish.

Northern blots. Total RNA was isolated from 5-day-old goldfish embryos and adult goldfish whole brain with the RNAzol B method (Biotecx Laboratories, Houston, USA) and poly A $^{+}-$RNA was selected with the Dynabeads mRNA purification Kit (Dynal, Oslo, Norway). A sample of $0.8 \mu \mathrm{g}$ poly $\mathrm{A}^{+}-\mathrm{RNA}$ was separated on a $1 \%$ agarose gel containing $1.1 \%$ formaldehyde, transferred to a Duralon UV nylon membrane (Stratagene, Heidelberg, Germany) and UV-crosslinked. Mcmbranes were prehybridized for $2 \mathrm{~h}$ at $50^{\circ} \mathrm{C}$ in hybridization solution ( $50 \%$ formamide, $10 \%$ dextran sulfate, $1 \%$ SDS, $1 M \mathrm{NaCl}, 100 \mu \mathrm{g} / \mathrm{ml}$ calf thymus DNA) followed by hybridization at $50^{\circ} \mathrm{C}$ overnight in hybridization solution containing a ${ }^{32} \mathrm{P}$-labeled antisense riboprobe. The blot was washed four times for $20 \mathrm{~min}$ each at $56^{\circ} \mathrm{C}$ in the following solutions: $2 \times \operatorname{SSC}(0.3 M$ sodium chloride, $0.03 M$ sodium citrate; $\mathrm{pH} 7) /$ $0.1 \%$ SDS; $1 \times \mathrm{SSC} / 0.1 \%$ SDS; $0.2 \times \mathrm{SSC} / 0.1 \% \mathrm{SDS}$, and $0.1 \times$ SSC/0.1\% SDS and exposed to X-ray film (Kodak X-OMAT 5AR, New York, USA).

An antisense ${ }^{32} \mathrm{P}$-labeled RNA probe was transcribed from the SP6 promotor of the TA cloning vector pCR II which contained the original 500 base pair PCR fragment (perE21.1) coding for the C-terminus of the neurolin protein. Onc microgram of linearized plasmid DNA served as a template for the generation of antisense riboprobes labeled with ${ }^{32} \mathrm{P}$ UTP $800 \mu \mathrm{Ci} / \mathrm{mmol}$ (Amcrsham, Braunschweig, Germany). Riboprobes were purified over a sephadex G50 column (Pharmacia, Freiburg, Germany).

Antibodies. Monoclonal antibodies were produced by injecting a 5-week-old Balb/c female mouse four times with immunopurified neurolin at two week intervals as follows: (1) intraperitoneally (i.p.) with the adjuvant ABM-2 complete (Sebak, Aidenbach, Germany); (2) i.p. with ABM-1 incomplete; (3) i.p. in $0.9 \% \mathrm{NaCl}$; (4) intravenously in $0.9 \% \mathrm{NaCl}$. Splecn cells were fused 3 days after the last immunization as previously described [21].

Polyclonal antibodies were produced by subcutaneously injecting rabbits with approximately $2 \mu \mathrm{g}$ of immunopurified ncurolin protein. The antigen was emulsified in Freund's adjuvant for the first injection and incomplete Freund's adjuvant for subsequent booster injections at 6,9 and 12 weeks. Serum samples werc collected 12 days after the third and fourth injections and stored at $-20^{\circ} \mathrm{C}$.

Immunoblots. Membranc fractions from adult zebrafish brains were isolated as previously described for goldfish [21]. Proteins were separated by $7.5 \%$ SDS-PAGE under non-reducing conditions on mini gels $6.5 \times 9 \mathrm{~cm}$ (Hoefer Scientific Instruments, Heidelberg). Proteins were transferred to Immobilon (Millipore, Bedford, Mass., USA) or Hybond-C Super (Amersham, Braunschweig, Germany), and membranes were blocked with $3 \%$ BSA (bovine serum albumin, fraction V; Sigma, Deisenhofen, Germany) in TBST (TRIS buffered saline plus $0.1 \%$ Tween). Western blotling was performed with the Vectastain Immunoperoxidase and DAB substrate Kit according to the manufacturer (Vector Laboratories, Burlingame, USA). Three antibodies against neurolin were tested on Western blots: the polyclonal antibodies 397 at a dilution of $1: 2000$ and $1: 4000$, the monoclonal antibody $441 / 3$ as undiluted supernatant or at a dilution of 1:10 and the original monoclonal E21 as super- 
natant or diluted $1: 10$ [21]. The biotinylated secondary antibody was diluted $1: 400$ in TBST.

Immunohistochemistry. Cryostat sections of the zebrafish retinotcctal system were prepared essentially as described previously for goldfish sections [21]. Staining was visualized using fluorescein isothiocyanate (FITC)-coupled secondary antibodies (Dianova, Hamburg, Germany).

\section{Results}

Isolation and nucleotide sequence of $c D N A s$ encoding goldfish and zebrafish neurolin

Degenerate PCR primers were synthesized based on amino acid sequence information obtained from the neurolin protein (see Methods). PCR using primers UL6 and UL21 and first strand cDNA from goldfish as a template, produced a 500 bp fragment (pcrE21.1) coding for the C-terminus of neurolin. The deduced amino acid sequence included an internal peptide sequence identical to that obtained from protein sequencing, and amino acids flanking the PCR primers (Fig. 1). A specific primer, UL22, was then synthesized based on the neurolin cDNA sequence and used together with the degenerate primer UL2, to amplify a $1.1 \mathrm{~kb}$ fragment (pcrE21.2) which overlapped with the pcrE21.1 fragment. The total cDNA sequence of $1569 \mathrm{bp}$ codes for an open reading frame (ORF) of 523 amino acids. All peptide sequences obtained from the goldfish neurolin protein were included in the amino acid sequence derived from this cDNA, indicating that we had cloned the cDNA for goldfish neurolin (Fig. 1).

The UL6 and UL21 primers and a cDNA template from embryonic zebrafish RNA were subsequently used in a PCR which produced a $500 \mathrm{bp}$ fragment coding for the C-terminus of zebrafish neurolin and corresponding to the pcrE21.1 fragment from goldfish. The sequence identity at the cDNA level in this region is $86 \%$. The predicted AA sequence is $88 \%$ identical $(95 \%$ similar) to goldfish neurolin indicating that it is most likely the zebrafish homolog (Fig. 3).

\section{Sequence analysis and comparison of neurolin to members of the immunoglobin superfamily}

The predicted neurolin amino acid sequence was compared with sequences in the Swisspir protein sequence database and was shown to be related to the chicken CAM DM-GRASP. At the amino acid level neurolin is $42 \%$ identical ( $51 \%$ similar) to the chick molecule DM-GRASP (Fig. 2). The human melanoma marker MUC18 [16] also shares some similarity with neurolin as do other members of the immunoglobulin (Ig) superfamily, although the homologies $(\leq 25 \%)$ are significantly lower than for DM-GRASP. For optimal alignment, nine gaps have been introduced into the comparison of neurolin and DM-GRASP (Fig. 2). The alignment demonstrates that the goldfish molecule is shorter than
MAP of Neurolin.seg

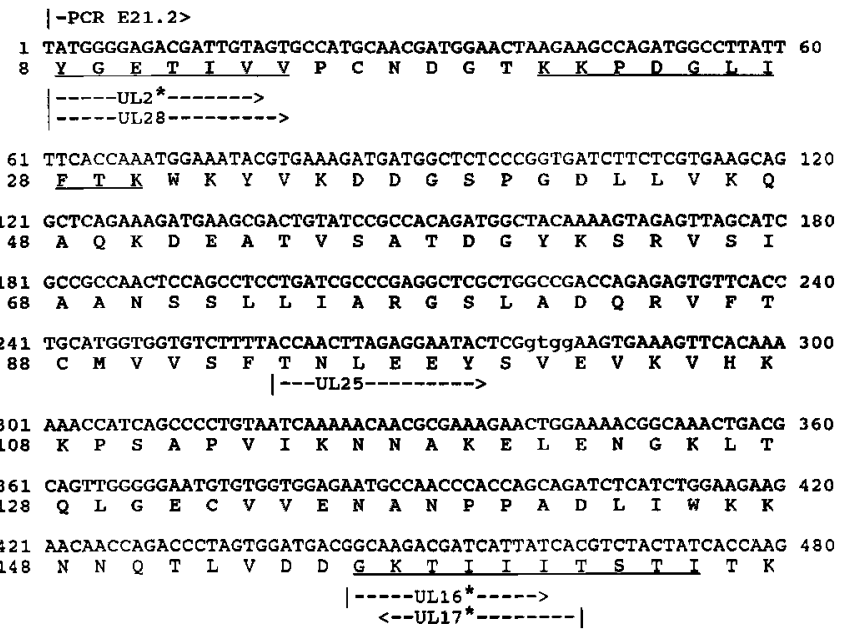

481 GACAAAATCACAGGTCTTTCCAGTACTTCCTCCCGTCTGCAGTACACAGCCAGGAAAGAG 540 $\begin{array}{llllllllllllllllllllll}168 & D & K & I & T & G & L & S & S & T & S & S & R & \text { L } & \text { Q } & \text { Y } & \text { T } & \text { A } & R & K & E\end{array}$ 541 GATGTAGAATCTCAGTTCACCTGCACAGCAAAGCATGTGATGGGCCCCGACCAGGTCTCA 600

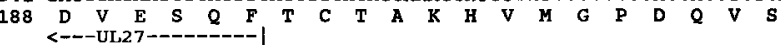

601 GAACCCGAGAGCTTCCCAATTCATTACCCCACCGAGAAGGTGAGTCTACAGGTTETTTCT 660

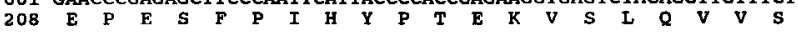
661 CAAAGCCCCATTAGGGAAGgCGAGGATGTGACACTGAAATGCCAGGCGGATGGAAACCCA 720 $\begin{array}{lllllllllllllllllllll}228 & Q & S & P & I & R & E & G & E & D & V & T & \text { L } & K & C & Q & A & D & G & N & P\end{array}$ 721 CCTCCTACCAGCTTCAACTTCAACATTAAGGGCAAAAAGGTCACGGTGACGGACAAGGAT 780 \begin{tabular}{llllllllllllllllllllllll}
248 & $\mathbf{P}$ & $\mathbf{P}$ & $\mathbf{T}$ & $\mathbf{S}$ & $\mathbf{F}$ & $\mathbf{M}$ & $\mathbf{F}$ & $\mathbf{N}$ & $\mathbf{I}$ & $\mathbf{K}$ & $\mathbf{G}$ & $\mathbf{K}$ & $\mathbf{K}$ & $\mathbf{V}$ & $\mathrm{T}$ & $\mathbf{V}$ & $\mathbf{T}$ & $\mathbf{D}$ & $\mathbf{K}$ & $\mathbf{D}$ \\
\hline
\end{tabular}

781 GTTTACACTCTGACTGGCGTTACCCGAGCAGACAGTGGCATTTACAAGTGCTCCCTGCTT 840

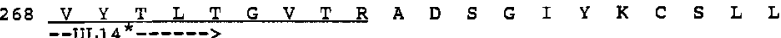

841 GACAACGATGTGATGGAGTCCACTCAGTTTGTCACCGTGAGCTTTCTGGATGTAAGCCTC 900 $\begin{array}{lllllllllllllllllllll}288 & D & N & D & V & M & E & S & T & Q & F & V & T & V & S & F & L & D & V & S & L\end{array}$

901 ACTCCTACAGGCAAGGTGTTAAAAATGTTGGGGAAACTTGATTGTGTCGTTGGATAAG 960 $\begin{array}{llllllllllllllllllllll}308 & T & P & T & G & & K & V & L & K & N & V & G & E & N & L & I & V & S & L & D & K\end{array}$

961 AACGCCTCTTCI'GAAGCAAAAGTGACATGGACTAAGGATAACCGTAAACTAGATAAACTG 1020

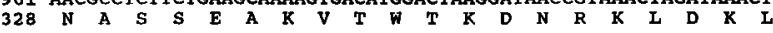

1021 CCCGACTTCTCCAAGTTGACATATAGTGATGCAGGCCTCTATGTCTGTGACGTGTCCATT 1080 $\begin{array}{lllllllllllllllllllll}348 & P & D^{2} & F & S & K & L & T & Y & S & D & A & G & L & Y & V & C & D & V & S & I\end{array}$ $<$ PCR E21.2 - I

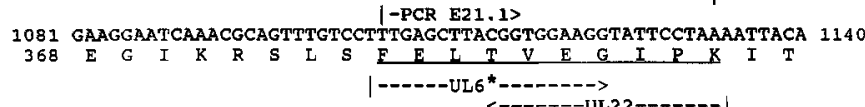

1141 AGTCTGACAAAGCATCGCAGCAGTGACGGCAAACACAAAGTCTTGACGTGTGAGGCAGAA 1200 388 S L T K $K$ H R S S D G K H K V L T C E A E 1201 GGTTCACCTAAACCTGACGTGCAGTGGAGTGTCAACGGAACTAACGATGAAGTGTCATAT 1260 $\begin{array}{llllllllllllllllllllll}408 & G & S & P & K & P & D & V & Q & W & S & V & N & G & T & N & D & E & V & S & Y\end{array}$

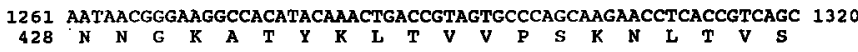
1321 TGTCTCGTGACCAATAAACTCGGTGAAGACACGAAGGAGATCAGCGTGTTTTCTCAGAAA 1380 $\begin{array}{lllllllllllllllllllllll}448 & C & L & V & T & N & K & \text { L } & G & E & D & T & K & E & I & S & V & F & S & Q & K\end{array}$ 1381 AACGAAGACGGCACCGAGCAGGCCAAAGTGATTGTGGGTATTGTGGTTGGGCTGCTTGTA 1440 $\begin{array}{lllllllllllllllllllll}468 & N & E & D & G & T & E & O & A & K & V & I & \nabla & G & I & \nabla & V & G & I & I & V\end{array}$ 1441 GCCGCTGCTITGGTGGGACTCATCTACTGGATCTATATCAAGAAAACAAGACAAGGCAGC $150^{\circ}$

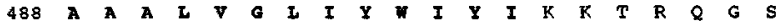

1501 TGGAAAACCGGCGAGAAAGAGGCAGGCACTTCAGAGGAGAGTAAGAAACTGGAGGAGAAT 1560

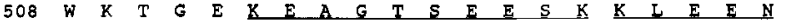
$<--$ UL22*-

1561 AATCACAAA 1569

$528 \stackrel{\mathrm{N} \quad \mathrm{H} \quad \mathrm{K}}{-----1}$

<PCR E21.1 -

Fig. 1. cDNA and predicted amino acid sequence of goldfish neurolin. The two overlapping polymerase chain reaction (PCR) fragments forming the neurolin clone are indicated above the DNA sequence. Peptide sequences determined by amino acid sequencing are underlined. Oligonucleotides used in PCR and sequencing are marked with $(\rightarrow)$ and degenerate primers with $(*)$. The putative transmembrane domain amino acid [(AA) 477-500] is in bold type 


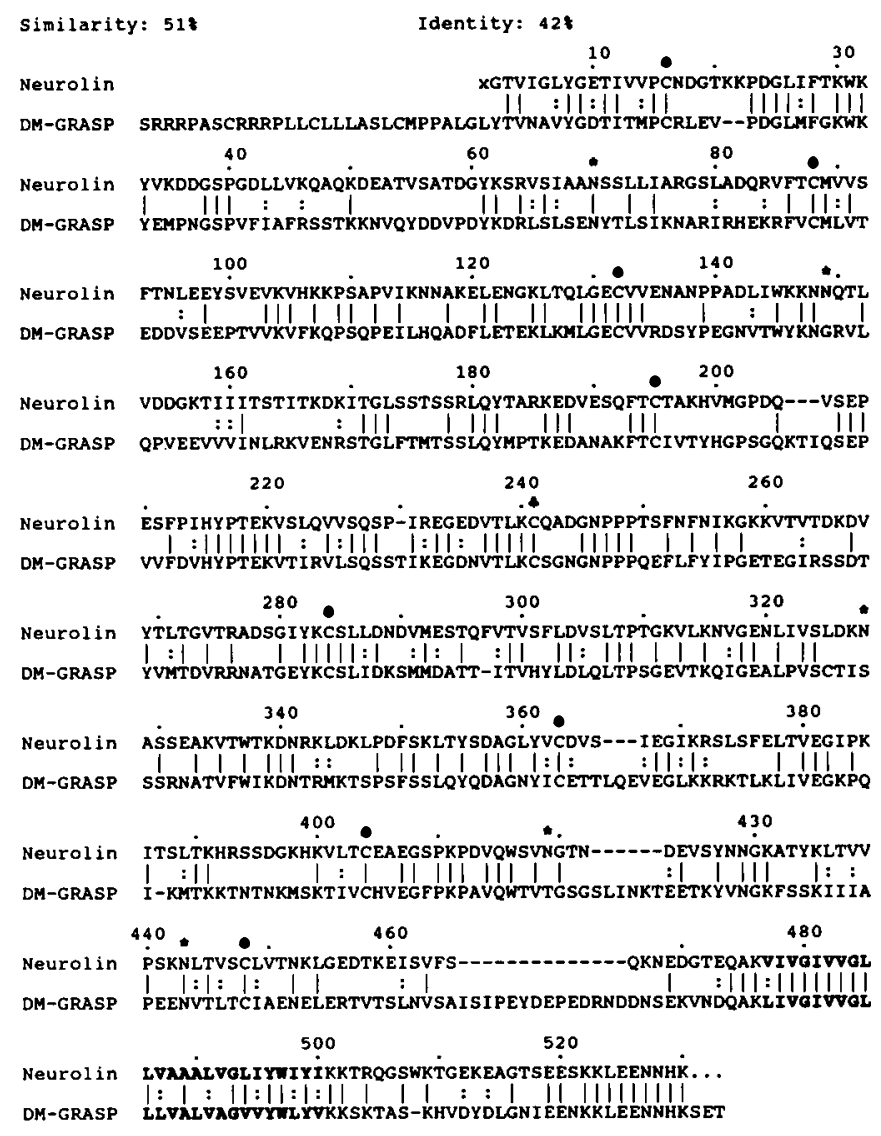

Fig. 2. Comparison of goldfish neurolin protein to chick DMGRASP. The predicted amino acid sequence of neurolin obtained from PCR clones aligned with the corresponding regions of the DM-GRASP protein. Identical amino acids are marked with a solid line and conservative exchanges are marked with a dotted line. The 9 cysteine (Cys) conserved in position in the two sequences are marked (\$). One Cys present in DM-GRASP is exchanged for a leucine in the goldfish sequence at AA position 325. Potential $\mathrm{N}$-linked glycosylation sites in the goldfish sequence are marked $\left({ }^{*}\right)$. The transmembrane domain (AA $477-500$ ) is in bold type

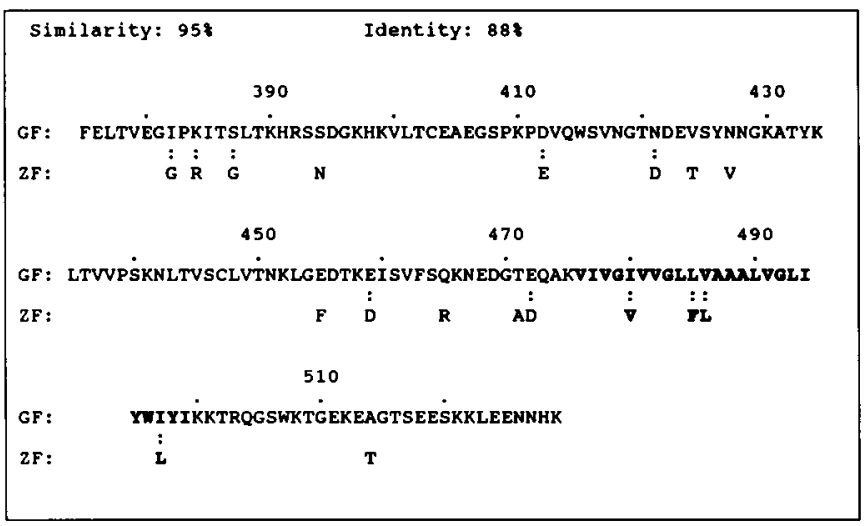

Fig. 3. Comparison of the amino acid sequences of goldfish $(G F)$ and zebrafish $(Z F)$ neurolin. The carboxy terminus of zebrafish neurolin obtained by PCR is compared with the corresponding region of goldfish neurolin (AA 376-530). Only amino acids differing from goldfish are shown in the zebrafish sequence. Conservative exchanges are indicated by a dotted line. The transmembrane domain is shown in bold letters

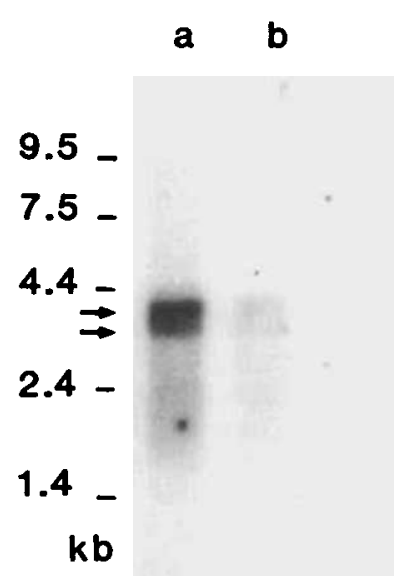

Fig. 4. Northern blot analysis of neurolin expression. Poly $\mathrm{A}^{+}$. RNA isolated from 5-day-old goldfish embryos (lane a) and whole brain of adult fish (lane $b$ ) was probed with a riboprobe transcribed from perE21.1 fragment coding for the carboxy terminus of neurolin

the chick protein. The identity between these molecules at the cDNA level, in the coding region, is $52 \%$.

This neurolin cDNA is incomplete, lacking sequences coding for eight amino acids at the $\mathrm{N}$-terminus based on amino acid sequencing, and part of the 3 '-end, including a stop codon. However, the molecular weight of neurolin on polyacrylamide gels, as well as the high conservation between neurolin and DM-GRASP at the C-terminus, suggests that only a short sequence ( $3 \mathrm{AA})$ is missing at the 3 '-end of the coding region in the neurolin cDNA. In addition, its sequence and cellular localization suggests that neurolin is an integral membrane protein. It is therefore expected, like DM-GRASP, to have a $\mathrm{N}$-terminal signal sequence.

Hydrophobicity blots predicted for neurolin indicate that it is a membrane protein having a large extracellular domain from AA 1-476, a short hydrophobic transmembrane region of $24 \mathrm{AA}$ and a short hydrophilic cytoplas. mic domain from AA 501-530. Four Ig superfamily type domains are predicted for neurolin between cysteine (Cys) AA position $16+88,132+195,241+284$ and $404+448$ (Fig. 2). A fifth Ig-domain was proposed for the protein DM-GRASP (AA $325+363$; see Discussion).

Glycosylation at one or more of the five potential $\mathrm{N}$-glycosylation sites present on the extracellular region of neurolin (Fig. 2) may account for the difference between the molecular weight predicted from the cDNA $(73 \mathrm{kDa})$ and the observed molecular weight of $80 \mathrm{kDa}$ on non-reducing polyacrylamide gels (under reducing conditions a molecular weight of $86 \mathrm{kDa}$ is observed). The neurolin protein was previously shown to bear an HNK-1/L2-epitope, typical for cell adhesion molecules [20], as well as several other carbohydrate epitopes [21].

\section{Neurolin $m R N A$ expression in goldfish}

Northern blots were used to analyze neurolin mRNA expression in 5-day-old goldfish embryos and adult 


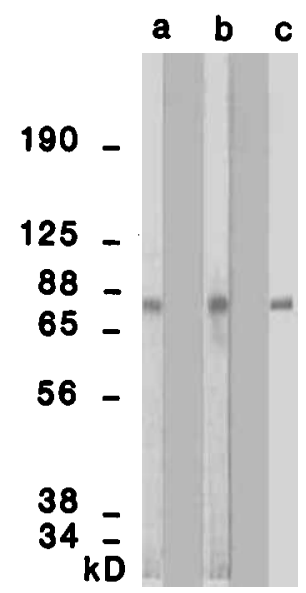

A

Fig. 5. A Western blot analysis of anti-neurolin antibodies (AB) on immunopurified goldfish neurolin. Lane $a$, original monoclonal $\mathrm{AB} \mathrm{E} 21$; lane $b$, monoclonal $\mathrm{AB} 441 / 3$; lane $c$, polyclonal $\mathrm{AB}$ 397. B Immunoblot of goldfish membrane octylglucoside (OG) extract. Lane a, AB 397; lane b, AB 441/3; lane $c, \mathrm{AB}$ E21. C Western blot analysis on zebrafish brain membrane $O G$ extract.

goldfish brain. As shown in Fig. 4 the pcrE21.1 riboprobe hybridizes to two bands, a major at $3.7 \mathrm{~kb}$ and an additional band at $3.3 \mathrm{~kb}$ in lanes containing either embryonic or adult brain mRNA. A much stronger hybridization signal for both bands is observed in embryos than is seen in adult brain, consistent with immunohistochemical studies. The doublet is clearly visible with shorter autoradiographic exposure times. A doublet on Northern blots was also reported for SC-1 [31] and BEN [22].

\section{Characterization of anti-neurolin antibodies on immunoblots}

Mono- (441/3) and polyclonal (397) antibodies were generated against the immunopurified goldfish neurolin protein. These antibodies were tested on immunoblots and compared to blots using the previously characterized monoclonal antibody E21 [21]. All antibodies recognize the affinity purified goldfish neurolin protein of $\approx 80 \mathrm{kDa}$ on non-reducing gels (Fig. 5A). An $80 \mathrm{kDa}$ band was also observed for all three antibodies on Western blots of membrane proteins from goldfish OG brain extract (Fig. 5B).

Immunoblots of zebrafish membrane proteins from brain OG extract also show staining of a protein at the same molecular weight as goldfish neurolin $(\approx 80 \mathrm{kDa})$ under non-reducing conditions, using both the monoclonal $441 / 3$ and polyclonal 397 antibodies (Fig. 5C), indicating that they most likely recognize the zebrafish equivalent of goldfish neurolin. The original monoclonal antibody E21 does not crossreact with zebrafish proteins.

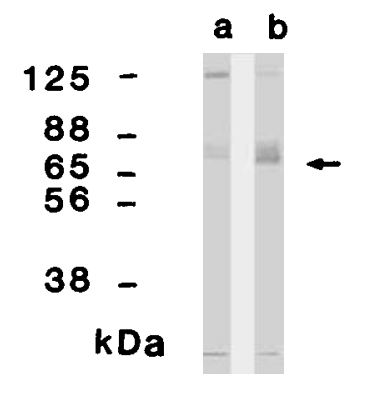

C

Lane $a, \mathrm{AB} 441 / 3$; lane $b, \mathrm{AB} 397$. All proteins were run without mercaptoethanol. An additional band appears on all OG brain blots at $\approx 120 \mathrm{kDa}$. This is unspecific staining and was observed only with the Vectastain Detection Kit. Control experiments subsequently done with the ECL Kit (Amersham, Braunschweig, Germany) do not show the additional band (data not shown)

\section{Neurolin expression in zebrafish}

The monoclonal antibody $441 / 3$ and the polyclonal antibodies 397 were used to determine the immunocytochemical localization of neurolin in adult and embryonic zebrafish retinotectal system.

A relatively old adult zebrafish ( 1.5 year) was chosen to examine whether neurolin staining in the zebrafish retina exhibits a pattern similar to that found in the adult goldfish retina where neurolin is observed only on RGCs at the margin and on new axons [21]. As demonstrated in Fig. 6a there were a few labeled cells in the RGC layer at the retinal periphery, but the number of such cells was noticeably lower than in goldfish of similar ages. Consistent with the small number of labeled RGCs, a comparably small group of labeled RGC axons was seen in cross sections through the retina (Fig. 6b). These labeled axons exit the eye and continue their path in the optic nerve (Fig. 6c). As previously described for goldfish [21], all other ganglion cell axons were unlabeled. This pattern was exhibited with both the monoclonal and polyclonal antibodies, although stronger staining was observed with the latter.

These antibodies were also applied to frozen sections of the adult zebrafish tectum (Fig. 7). There was diffuse staining over most of the tectum but as in goldfish tectum [21], labeling was pronounced in three bands in the synaptic layers of RGC axons, stratum fibrosum et griseum superficiale (SFGS), but was absent from the retinal axon fascicles in the stratum opticum (SO). The most superficial layer of the the tectum, stratum marginale (SM), was entirely bare of labeling but bright staining was observed in the meninges. A band of bright labeling was noted in the stratum griseum centrale (SGC), and may correlate with the small subpopulation of RGC 

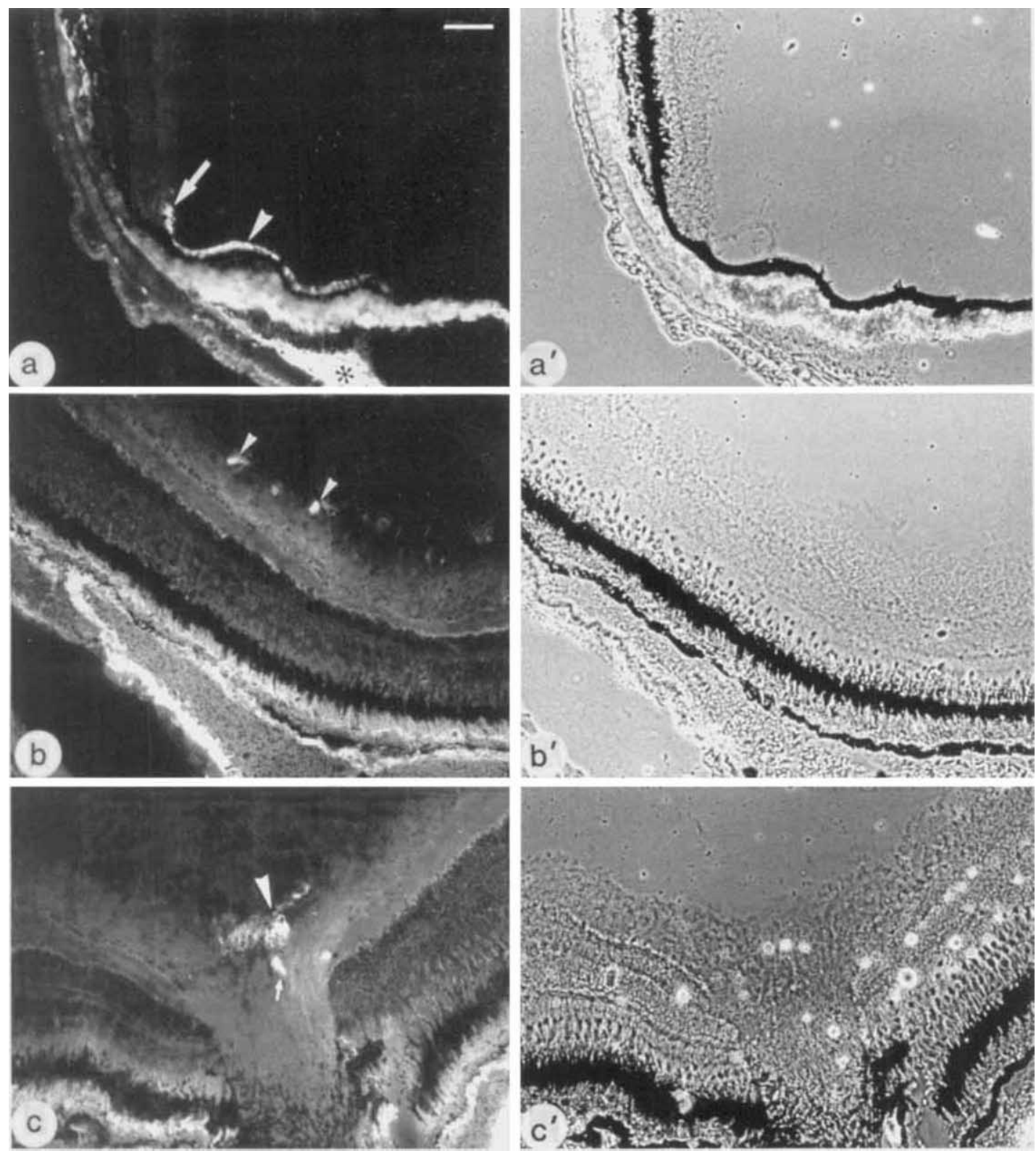

Fig. 6. Cross sections through the retina of an adult zebrafish. a. b, c Immunofluorescent images; $\mathbf{a}^{\prime}, \mathbf{b}^{\prime}, \mathbf{c}^{\prime}$, corresponding images in phase contrast. a The polyclonal antibodies 397 against purified goldfish neurolin recognize new retinal ganglion cells (RGCs) at the margin in the adult zebrafish retina (arrow). Staining extends also to the iris (arrowhead) and epithelia $\left({ }^{*}\right)$ outside the retinal

axon terminals known to reside in this position $[17,28]$. Bright staining was also seen in the layer stratum album centrale (SAC) of the zebrafish tectum when tectal efferents joined in fascicles pass through the tectum and this staining extends to where these axons leave the tectum. In the stratum periventriculare (SPV), where most of the somata of tectal neurons with long apical dendrites and radial glial cells are accumulated $[17,26]$, labeling was seen around some clustered cells. These cells extend processes radially through the tectum. In addition, a few processes in radial orientation exhibited staining (Fig. 7). This was unique to zebrafish and was not seen in margin. b Bundles of new axons (arrows) derived from the labeled RGCs are stained. All other RGCs and axons, as well as the remaining neurons and cells of the retina are unlabeled. $c$ The new labeled RGC axons gather at the optic disc (arrowhead) and are seen to exit the eye as a fascicle through the optic nerve (arrow). Bar, $50 \mu \mathrm{m}$

goldfish tectum [21]. It is not clear at present whether the processes originate from these labeled cell clusters and whether they are of neuronal or glial origin.

The antibodies $441 / 3$ and 397 were tested on sections of zebrafish embryos to assess whether they could be used for future studies detailing the regulation of neurolin during embryonic development. Figure 8 shows a cross section through a $72 \mathrm{~h}$ PF zebrafish brain including the eyes and the optic nerve stained with the polyclonal antibodies 397 . In contrast to adult fish, all RGCs were labeled in embryos. Those close to the margin were more strongly stained than the RGCs in the central reti- 


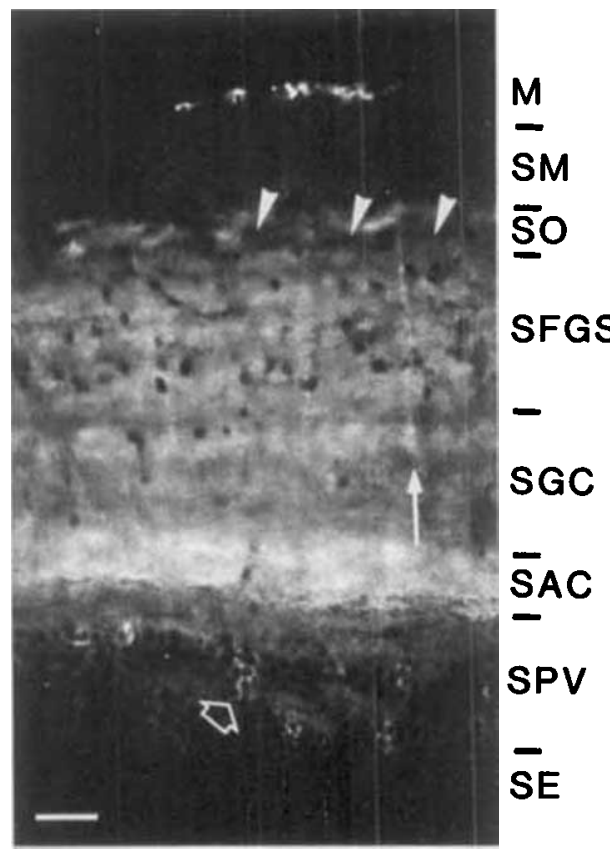

Fig. 7. Cross section through the tectum of an adult zebrafish. Using the polyclonal antibodies 397 weak staining is seen throughout the tectal layers SFGS to SAC, while the SM is unlabeled. The retinal axon fascicles (arrowheads) in the $\mathrm{SO}$ are also unlabeled. Most brightly stained are three bands of the retinal axon terminal arbor layers SFGS, a band in the SGC, perhaps correlating with the few retinal axons in this layer, and the tectal efferent axons in the SAC. Labeled cells in the SPV occur in small clusters, and occasional radial processes (arrow) (perhaps emerging from the labeled cells) are also brightly labeled. Further staining occurs in the meninges. $M$, meninges; $S M$, stratum marginale; $S O$, stratum opticum; $S F G S$, stratum fibrosum et griseum superficiale; $S G C$, stratum griseum centrale; $S A C$, stratum album centrale; $S P V$, stratum periventriculare. $B a r, 25 \mu \mathrm{m}$

na, a phenomenon not noted in goldfish embryos [21]. Staining extended to the retinal axons in the retina and was visible on all axons exiting the retina and in the optic nerve.

Labeling in the spinal cord of zebrafish embryos is similar to that previously described for goldfish embryos [21], including the ventral floor plate cells, cells in a position of motoneurons, the dorsal roots and their entry zone and fiber tracts running dorsally in the spinal cord (data not shown).

\section{Discussion}

Neurolin was previously identified as a cell surface glycoprotein expressed on embryonic and regenerating axons in the goldfish visual system [21]. We report here the sequence of a cDNA coding for goldfish neurolin and a cDNA coding for the carboxy terminus of this molecule in zebrafish. The predicted amino acid sequence of neurolin suggests that it is a member of the Ig superfamily with similarities to the chick CAM DMGRASP $[2,22,31]$. In addition, we have generated crossreacting anti-neurolin antibodies to study neurolin expression in zebrafish. Immunohistochemical studies in
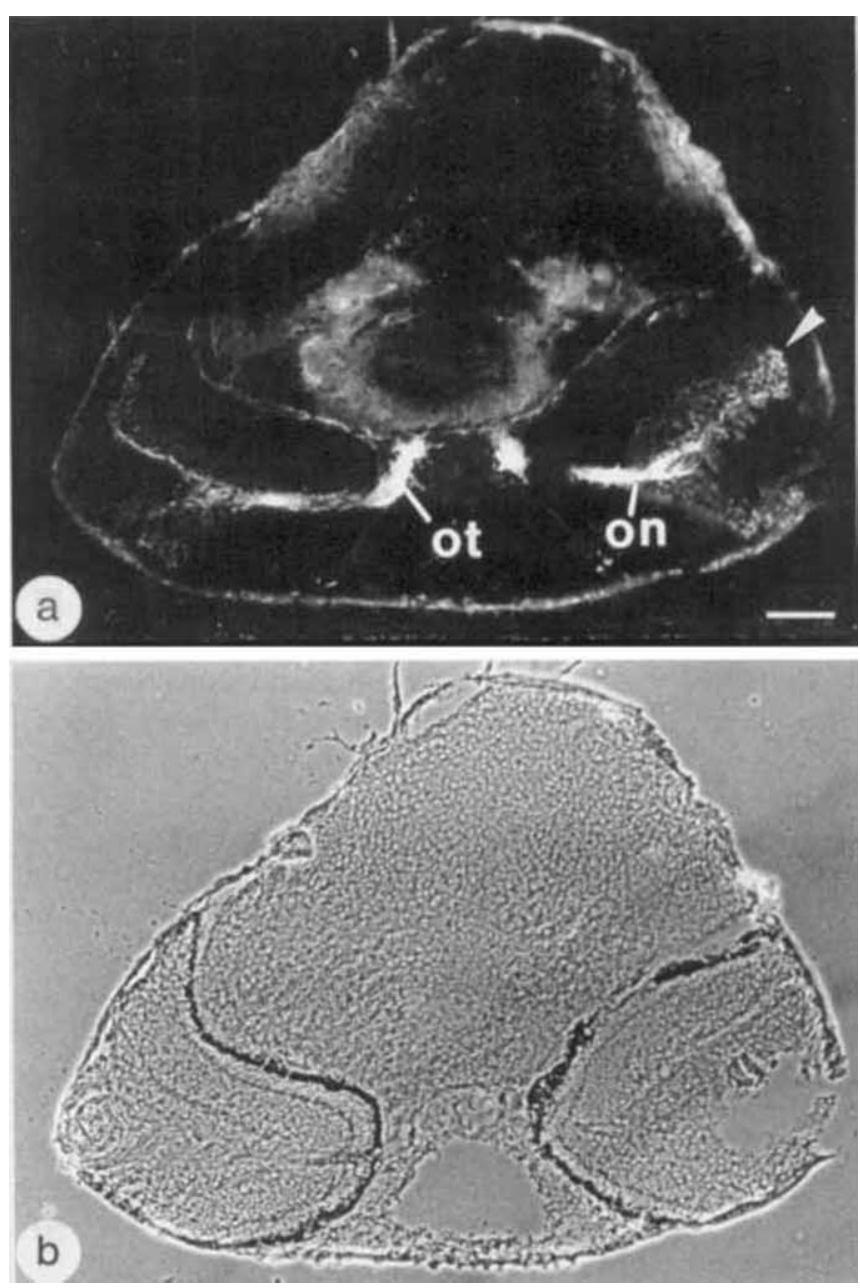

Fig. 8. Cross section through the head of a 72 hours postfertilization (h PF) zebrafish embryo: immunofluorescence image (a); in phase contrast (b). a The polyclonal antibodies 397 recognize the RGCs and their axons in zebrafish embryos. The RGCs at the margin (arrowhead) are labeled more heavily than the central RGCs. Staining extends to the retinal axons, seen here as they leave the eye, and enter the optic nerve (on), and in the proximal portion of their ascent to the tectum in the optic tract $(o t)$. The epithelia around the head are also labeled, as well as an unidentified structure between the eyes. Dorsal is at the top. Bar, $50 \mu \mathrm{m}$

the zebrafish retinotectal system reveal a similar distribution to that described earlier in goldfish [21]. Furthermore, preliminary experiments in embryonic zebrafish - a model system for developmental studies in vertebrates - indicate that neurolin expression is developmentally regulated in the visual system. Developmental and functional studies investigating the role of neurolin in axonal growth and regeneration will now be possible.

Analysis of the goldfish neurolin cDNA suggests that the predicted protein is capable of forming Ig domains. These domains are a common structure among several CAMs (for review see [33]), many of which are expressed in the developing nervous system [8,13]. A comparison of fish neurolin and chick DM-GRASP shows conservation in regions surrounding Cys residues. High conservation between these molecules is seen at the carboxy-terminus representing the putative transmembrane $(88 \%)$ 
and cytoplasmic domains $(66 \%)$ suggesting that the functions of these domains may be conserved. The extracellular domain of neurolin, however, is significantly shorter (23 AA) than that of DM-GRASP and shares only a $45 \%$ similarity.

Although the overall structure of these molecules is well conserved, there is a noticeable difference. Neurolin has 9 Cys residues with the possibility of forming $4 \mathrm{Ig}$ loops, whereas DM-GRASP has 10 Cys residues and has been suggested to have $5 \mathrm{Ig}$ domains, $2 \mathrm{~N}$-terminal V-type domains, followed by 3 C-type domains [2, 33]. Neurolin, however, has the hydrophobic AA leucine in a position corresponding to Cys residue 325 in DMGRASP of the fourth Ig domain (Fig. 2). Other molecules belonging to the Ig superfamily, such as the PDGFR, were shown to lack Cys residues in possible Ig domains and have instead hydrophobic amino acids [33]. It should be noted that this region between the two Cys in DM-GRASP is shorter than is typical for C-type Ig domains [33]. It will be interesting to see whether such substitutions appear more frequently in fish than in higher vertebrates. To our knowledge, there is no other CAM sequence information available in fish at this time. Comparisons of other Ig-like CAMs that have been cloned in more than one species $[3,9,11$, $19,23,34]$ show higher conservation between these molecules in vertebrate species than is seen here between fish neurolin and chick DM-GRASP. For example, the sequence of N-CAM in Xenopus [15] and chick [12] is $75 \%$ identical at the AA level. Neurolin appears fairly well conserved between zebrafish and goldfish having an $88 \%$ identity $(95 \%$ similarity) at the AA level for the region we have compared.

It is not clear from this analysis if neurolin is the fish equivalent of chick DM-GRASP or a related family member. However, we were unable to find a molecule more conserved to DM-GRASP in both zebrafish and goldfish using PCR. In addition, we screened several goldfish cDNA libraries with a chick DM-GRASP cDNA probe [2] and also failed to detect a molecule more closely related to DM-GRASP. As has been suggested, the conserved structure of Ig domains may be functionally important for mediating cell adhesion [33]. Therefore, the structural conservation between the chick and goldfish molecules indicate that they may be functional homologs although the overall sequence identity is low $(42 \%)$.

In previously published studies there was no report of DM-GRASP, SC-1 or BEN expression in the chick visual system $[2,22,31]$. However, an antibody generated independently against DM-GRASP does show staining in the embryonic chick visual system (E. Pollerberg, personal communication). All the above chick antibodies label strongly in the chick spinal cord, where they recognize the ventral floor plate, motoneurons, dorsal root and the dorsal funiculus $[2,22,30,31]$. A comparable staining pattern was found with the anti-neurolin antibodies in the embryonic goldfish [21] and zebrafish spinal cord (data not shown).

In normal adult goldfish, only RGCs at the retinal margin were labeled with anti-neurolin antibodies. Their position suggests that they are new ganglion cells [14]. These cells were, by far, less numerous in adult zebrafish than in adult goldfish, suggesting that the goldfish retina continues to grow more vigorously than that of zebrafish. This might have been expected since goldfish are known to increase their overall size over years, and concurrently add new neurons to their retinae [14] and tecta [18]. While zebrafish enlarge in size during their larval development, they cease to grow noticeably at about 6 months when they begin to breed. The limited number of labeled ganglion cells in zebrafish correlates with the equally small population of labeled retinal axons in the eye and optic nerve. In zebrafish embryos the antibodies recognize all ganglion cells and retinal axons on their path to the tectum. This pattern is similar to that observed in goldfish embryos with the original monoclonal antibody E21 and with the present antibodies. However, the peripheral (youngest) RGCs were stained more brightly than the more central (older) ones. The immunoblot data, in conjunction with the immunohistochemical staining in zebrafish and goldfish, strongly suggest that the monoclonal $441 / 3$ and the polyclonal antibodies 397 recognize the zebrafish equivalent to goldfish neurolin.

From its presence on embryonic RGC axons, on new RGC axons in the adult, and on regenerating retinal axons after ONT, we hypothesize that neurolin is important during retinal axonal growth. However, it apparently co-exists with a number of other cell surface molecules, which we called growth-associated cell surface molecules. Two of these molecules were identified as NCAM 180/140 [1] and E 587-antigen [32], a member of the L1 family of cell adhesion molecules. The protein sequence similarities between neurolin and the CAM DM-GRASP, suggests that neurolin belongs to the same functional category. It is not understood why growing axons co-express several CAMs simultaneously or whether each molecule serves a specific function. However, at no time after ONT did we observe all regenerating axons to be labeled by the E21 antibody. In contrast, NCAM 180/140 [1] and the E 587 antigen [32] were shown to be on all regenerating axons over a period of weeks. This suggests that only a subpopulation of axons express neurolin or that the temporal window of neurolin expression is more narrow. If neurolin expression on retinal axons is found during the early stages of axonal outgrowth, this would imply that neurolin is important for retinal axons during initial phases of growth cone extension, as has been suggested for TAG-1 $[4,9]$. Future studies combined with in situ hybridization techniques are designed to study the regulation of neurolin expression and to elucidate its function during axonal growth and regeneration in fish.

Acknowledgements. The authors wish to thank Suzannah Chang for providing us a chick cDNA clone of DM-GRASP. We also thank Marianne Wiechers for her excellent technical assistance in purifying the neurolin protein, and all our colleagues who helped with the isolation of goldfish brains. Anette-Yvonne Loos observed the zebrafish breeding colony, and Ulrich Weiland helped with sectioning and immunostaining of the zebrafish brains, and we thank them for their help. We thank Jan Weiler in the group of 
Prof. Dr. W. Pfleiderer, Faculty of Chemistry, University of Konstanz, for the oligonucleotide synthesis. Also we would like to thank Mary Anne Cahill for helping with the illustrations and Dr. Rupert Mutzel and Dr. Michael Ehrmann for carefully reading the manuscript. Suzanne Giordano is supported by the HumboldtStiftung. This work was supported by grants of the DFG, SFB 156, TPC 6 to C.A.O.S.

\section{References}

1. Bastmeyer M, Schlosshauer B, Stuermer CAO (1990) The spatiotemporal distribution of N-CAM in the retinotectal pathway of adult goldfish detected by the monoclonal antibody D3. Devclopment 108:299-311

2. Burns FR, Kannen S von, Guy L, Raper JA, Kamholz J, Chang S (1991) DM-GRASP, a novel immunoglobulin superfamily axonal surface protcin that supports neurite extension. Neuron 7:209-220

3. Cunningham BA, Hemperly JJ, Murray BA, Prediger EA, Brackenbury R, Edelman GM (1987) Neural cell adhesion molecule: Structure, immunoglobulin-like domains, cell surface modulation, and alternative RNA splicing. Science 236:799806

4. Dodd J, Morton SB, Karagogeos D, Yamamoto M, Jessell TM (1988) Spatial regulation of axonal glycoprotein expression of subsets of embryonic spinal neurons. Neuron 1:105-116

5. Doherty P, Walsh FS (1992) Cell adhesion molecules, second messenger and axonal growth. Curr Opin Neurobiol 2:595-601

6. Eckerskorn C, Lottspeich F (1989) Internal amino-acid sequence-analysis of proteins separated by gel-electrophoresis after tryptic digestion in polyacrylamide matrix. Chromatographia 28:92-94

7. Eckerskorn C, Mewes W, Goretzki H, Lottspeich F (1988) A new siliconized glass fiber as support for protein-chemical analysis of electroblotted proteins. Eur J Biochem 176:509-519

8. Edelman GM, Crossin KL (1991) Cell adhesion molecules: Implications for a molecular histology. Annu Rev Biochem 60:155-190

9. Furley AJ, Morton SB, Manalo D, Karagogeos D, Dodd J, Jessell TM (1990) The axonal glycoprotein TAG-1 is an immunoglobulin superfamily member with neurite outgrowth-promoting activity. Cell $61: 157-170$

10. Gaze RM (1970) The formation of nerve connections. Academic Press, New York, pp 118-171

11. Harper JR, Prince JT, Healy PA, Stuart JK, Nauman SJ, Stallcup WB (1991) Isolation and sequence of partial cDNA clones of human L1: Homology of human and rodent L1 in the cytoplasmic region. $J$ Neurochem 56:797-804

12. Hemperley JJ, Edelman GM, Cunningham BA (1986) cDNA clones of the neural cell adhesion molecule (N-CAM) lacking a membrane-spanning region consistent with evidence for membrane attachment via a phosphatidylinositol intermediate. Proc Natl Acad Sci USA 83:9822-9826

13. Jessel TM (1988) Adhesion molecules and the hierarchy of neural development. Neuron 1:3-13

14. Johns PA (1977) Growth of the adult goldfish eye: III. Source of the new retinal cells. J Comp Neurol 176:343-357

15. Krieg PA, Sakaguchi DS, Kintner CR (1989) Primary structure and development expression of a large cytoplasmic domain form of Xenopus laevis neural cell adhesion molecule (NCAM). Nucleic Acids Res 17:10321-10335

16. Lehmann JM, Riethmueller G, Johnson JP (1989) MUC18, a marker of tumor progression in human melanoma, shows sequence similarity to the neutral cell adhesion molecules of the immunoglobulin superfamily. Proc Natl Acad Sci USA $86: 9891-9895$
17. Meek $J$ (1983) Functional anatomy of the tectum mesencephali of the goldfish: An explorative analysis of the functional implications of the laminar structural organization of the tectum. Brain Res Rev 6:247-297

18. Meyer RL (1978) Evidence from thymidinc labeling for continuing growth of retina and tectum in juvenile goldfish. Exp Neurol 59:99-111

19. Moos M, Tacke R, Scherer H, Teplow D, Frueh K, Schachner M (1988) Neural adhesion molecule L1 as a member of the immunoglobulin superfamily with binding domains similar to fibronectin. Nature 334:701-703

20. Naronha AB, Illyas A, Antonicck H, Schachner M, Quarles RH (1986) Molecular specificity of L2 monoclonal antibodies that bind to carbohydrate determinants of neural cell adhesion molecules and their resemblance to other monocolonal antibodies recognizing the myelin-associated glycoprotein. Brain Res 385:237-244

21. Paschke KA, Lottspeich F, Stuermer CAO (1992) Neurolin, a cell surface glycoprotein on growing retinal axons in the goldfish visual system, is reexpressed during retinal axonal regeneration. J Cell Biol 117:863-875

22. Pourquié O, Corbel C, LeCaer J-P, Rossier J, LeDouarin NM (1992) BEN, a surface glycoprotein of the immunoglobulin superfamily, is expressed in a variety of devcloping systems. Proc Natl Acad Sci USA 89:5261-5265

23. Prince JT, Alberti L, Healy PA, Naumann SJ, Stallcup WB (1991) Molecular cloning of NILE glycoprotein and evidence for its continued expression in mature rat CNS. J Neurosci Res 30:567-581

24. Sanger F, Nicklen S, Couson AR (1977) DNA sequencing with chain-terminating inhibitors. Proc Natl Acad Sci USA $74: 5463-5467$

25. Skene JHP (1989) Axonal growth-associated proteins. Annu Rev Neurosci 12:127-156

26. Stevenson JA, Yoon MG (1982) Morphology of radial glia. ependymal cells, and periventricular neurons in the optic tectum of goldfish (Carassius auratus). J Comp Neurol 205:128-138

27. Stuermer CAO (1988) Retinotopic organization of the developing retinotectal projection in the zebrafish embryo. I Neurosci $8: 4513-4530$

28. Stuermer CAO, Easter SS (1984) A comparison of the normal and regenerated retinotectal pathway of goldfish. J Comp Neurol $223: 57-76$

29. Stuermer CAO, Bastmeyer M, Baehr M, Strobel G, Paschke $\mathrm{K}$ (1992) Trying to understand axonal regeneration in the CNS of fish. J Neurobiol 23:537-550

30. Tanaka H, Obata K (1984) Developmental changes in unique cell surface antigens of chick embryo spinal motoneurons and ganglion cells. Dev Biol 106:26-37

31. Tanaka H, Matsui T, Agata A, Tomura M, Kubota I, McFarland KC, Kohr B, Lee A, Phillips HS, Shelton DL L(1991) Molecular cloning and expression of a novel adhesions molecule, SC1. Neuron 7:535-545

32. Vielmetter J, Lottspeich F, Stuermer CAO (1991) The monoclonal antibody E587 recognizes growing (new and regenerating) retinal axons in the goldfish retinotectal pathway. $J$ Neurosci $11: 3581-3593$

33. Williams AF, Barclay AN (1988) The immunoglobulin superfamily - domains for cell surface recognition. Annu Rev Immunol $6: 381-405$

34. Zuellig RA, Rader C, Schroeder A, Kalousek MB, Van Bohlen, Halbach F, Osterwalder T, Inan C, Stoeckli AT, Affolter HU, Fritz A, Hafen E, Sonderegger P (1991) The axonally secreted cell adhesion molecule, Axonin-1: Primary structure, immunoglobulin-like and fibronectin-type-III-like domains and glycosyl-phosphatidylinositol anchorage. Eur J Biochem 204:453463 